\title{
«Voyez ce que je vois!»
}

Peter Bertchtolda, Urs Zanonib

${ }^{a} \mathrm{PD} \mathrm{Dr},{ }^{b} \mathrm{MPH}, \mathrm{fmc}-$ le forum suisse des soins intégrés

Les impressionnantes innovations dans la technologie médicale des dernières années sont dans l'intérêt de nombreux patients. Dans le même temps, elles fragmentent les chemins thérapeutiques et génèrent des lacunes qualitatives. C'est précisément à cet endroit que la perspective du patient revêt une signification particulière, car ce sont essentiellement les patients et leurs proches qui ont une vue d'ensemble du processus thérapeutique. De nouveaux travaux de recherche montrent le lien étroit entre la focalisation sur le patient, les soins intégrés et la qualité.

"At a time when anger and distrust seem ubiquitous in the health systems of so many countries, asking patients to report on the quality of their care may bring clinicians and those they serve closer together.» (Prof. Dr Thomas Delbanco)

\section{Introduction}

Publiée dans le British Medical Journal en 1996, cette citation de Thomas Delbanco, professeur à Harvard, ne pourrait être plus actuelle: si le traitement doit vraiment être focalisé sur le patient, les soins doivent être bien davantage envisagés et planifiés à travers les yeux des patients qu'ils ne le sont à présent [1]*. En effet, les possibilités thérapeutiques sont à présent largement plus nombreuses, principalement grâce aux progrès médicaux et technologiques: à l'avantage des patients car plus de maladies sont aujourd'hui traitées avec succès et les souffrances atténuées de manière plus efficace. Mais paradoxalement au détriment des mêmes patients car ces avancées se répartissent inévitablement sur plus d'acteurs, ce qui fragmente davantage le parcours thérapeutique et génère des lacunes qualitatives.

Pour le patient, cette évolution implique un nombre croissant de professionnels et d'interlocuteurs coresponsables. Ceci a pour effet d'accroître la distance entre ces derniers et leurs patients, lesquels se sentent toujours plus désorientés et perdus dans une masse d'informations. Cette perception est particulièrement fréquente chez les malades chroniques souffrant de plusieurs pathologies dont le parcours de soins est long [2].

\footnotetext{
* Les références se trouvent sous www.bullmed.ch $\rightarrow$ Numéro actuel ou $\rightarrow$
} Archives $\rightarrow 2015 \rightarrow 14-15$. avant tout les patients (et éventuellement leurs proches) qui disposent d'un point de vue privilégié sur l'ensemble des fragments individuels de leur traitement, et dès lors sur le processus thérapeutique dans son intégralité. Rien ni personne n'est en mesure de remplacer cette expérience.

Il est donc clair que le point de vue du patient constitue une source essentielle lorsqu'il s'agit d'observer et d'optimiser les processus thérapeutiques et leur qualité. Tandis que les professionnels de tous les secteurs se consacrent inévitablement à un seul fragment thérapeutique, les patients et leurs proches vivent toujours le processus de prise en charge dans son intégralité.

$\mathrm{Au}$ fil des dernières années, ce constat a suscité de nombreuses initiatives et projets dans le but d'imbriquer plus étroitement la perspective du patient avec son traitement, et de promouvoir ce faisant des soins véritablement focalisés sur le patient. Citons par exemple l'Université de Heidelberg qui a développé et validé le premier questionnaire permettant d'identifier les lacunes qualitatives dans un processus thérapeutique [3]. Un autre exemple est celui du Department of Health britannique qui a publié le document stratégique "No decision about me, without me» en nourrissant l'intention d'intégrer les patients de manière plus directe dans leur traitement impliquant une prise de décision partagée [4].

\section{... et soins intégrés}

Il n'est dès lors pas surprenant que les personnes concernées évoquent rapidement la coordination, l'interconnexion et la continuité des soins lorsqu'elles sont interrogées au sujet du traitement focalisé sur le patient. Les patients savent très bien ce que ces prin- 
cipes signifient pour leur traitement et ressentent rapidement leur défaut [5]. De nouveaux travaux de recherche indiquent également un lien étroit entre la focalisation sur le patient et le traitement interconnecté (soins intégrés) [6]. Cette mise en exergue s'opère à juste titre, car pour un patient ayant une vue sur l'ensemble de son anamnèse et son plan thérapeutique, les fragmentations et interruptions sont visibles et vécus comme un problème. Dès lors, il est presque inévitable que d'éventuelles lacunes qualitatives se manifestent pour lui surtout aux interfaces [7].

\section{La focalisation sur le patient de manière concrète}

A vrai dire, que signifie la focalisation sur le patient dans le domaine des soins? Comment les patients peuvent-ils être davantage impliqués dans le processus décisionnel tout au long des chemins thérapeutiques? Par le passé, les discussions et publications relatives à ces questions furent déjà très nombreuses, si bien que la focalisation sur le patient suscitait souvent davan-

Les patients savent très bien ce que la coordination des soins signifie pour leur traitement et ressentent rapidement leur défaut.

tage de hochements de tête que d'intérêt, voire des discussions plutôt moqueuses que sérieuses. Ceci n'est guère étonnant dans la mesure où la plupart de ces discussions se tiennent au sujet des patients mais presque jamais avec eux.

De très récents résultats de recherche permettent d'opérationnaliser le concept abstrait de «focalisation sur le patient» selon quatre dimensions:

1. L'information compréhensible des patients, ce qui signifie que les patients et leurs proches reçoivent suffisamment d'informations quant au processus thérapeutique dans sa totalité afin qu'ils puissent se faire une idée de son déroulement, du résultat et des risques;

2. Implication des patients dans la prise de décisions, ce qui signifie que les patients donnent non seulement leur consentement à une recommandation de traitement (dans le sens du "consentement éclairé») mais connaissent les autres possibilités de traitement éventuelles et/ou le soignant leur recommande un deuxième avis;

3. Coordination et continuité du traitement et de la prise en charge, ce qui signifie que les professionnels qui assurent la prise en charge évaluent les résultats d'examens de manière uniforme et en tirent des conclusions concordantes destinées au patient et à ses proches en termes de diagnostic, de traitement et de pronostic;

4. Communication cohérente entre les prestataires, ce qui signifie que les professionnels qui assurent la prise en charge connaissent leurs rôles et fonctions respectifs, assurent ensemble une prise en charge optimale et respectent en particulier les interlocuteurs directs des patients et de leurs proches.

Ces résultats de recherche illustrent de manière im pressionnante ce qui est pertinent du point de vue du patient ainsi que la façon dont la qualité dépend sous toutes ses facettes d'une imbrication bien huilée de toutes les institutions et des professionnels impliqués. "Voyez ce que je vois!» est une invitation aux professionnels à tenir compte de la vision globale du patient dans la planification des différents fragments de sa prise en charge. C'est en même temps de l'opportunité d'aborder les revendications parfois irréalistes ou excessives des personnes concernées.
Correspondance: PD Dr Peter Berchtold Forum Managed Care Zugerstrasse 193 CH-6314 Neuäger peter.berchtold[at]fmc.ch

\section{Symposium national des soins intégrés le 24 juin 2015 en la Kursaal de Berne}

Sous le titre «De la perspective du patient vers plus d'intégration", le symposium national 2015 du Forum suisse pour les soins intégrés fmc met en lumière la perspective du patient comme source essentielle afin de mieux harmoniser les différentes étapes thérapeutiques. Des personnalités issues du secteur de la santé suisse lanceront des pistes de réflexion concernant la possible utilisation de la perspective du patient. Ils évoqueront les mesures d'encouragement permettant d'améliorer l'intégration et, dès lors, la qualité des soins. "Davantage de discussion pour plus d'utilité» est le deuxième axe essentiel du symposium: les participants dévoileront leurs perspectives et expériences durant un débat dont l'encadrement est assuré par un animateur. Le symposium aura lieu à Berne avec traduction simultanée en français et en allemand. Vous trouverez de plus amples informations sur le symposium fmc 2015 sur www.fmc.ch

Le symposium 2015 est reconnu par I'Institut suisse pour la formation médicale postgraduée et continue (ISFM) avec 5 crédits. 


\section{Références}

1 Delbanco TL. Quality of care through the patient's eyes. Brit Med J. 1996;313:832-3.

2 Barry JB. Shared Decision Making - The Pinnacle of Patient Centered Care. New Engl J Med. 2012;366: 780-1.

3 Noest S, Ludt S, Klingenberg A, Glassen K, Heiss F, Ose D, Rochon J, Bozorgmehr K, Eensing M, Szecseny J. Involving patients in detecting quality gaps in a fragmented healthcare system: development of a questionnaire for Patients' Experiences Across Health Care Sectors. Int J Qual Health Care. 2014;26:240-9.

4 NHS Department of Health. Liberating the NHS: No decision about me, without me. 2012. www.gov.uk/government/uploads/ system/uploads/attachment_data/file/21698o/Liberatingthe-NHS-No-decision-about-me-without-me-Governmentresponse.pdf
5 Walker KO, Labat A, Choi J, Schmittdiel J, Stewart AL, Grumbach K. Patient perceptions of integrated care: confused by the term, clear on the concept. Int J Integr Care. 2013;Jan-Mar.

6 Juhnke C, Mühlbacher A. Patient-centeredness in integrated healthcare delivery systems - needs, expectations and priorities for organised healthcare systems. Int J Integr Care. 2013; Oct-Dec.

7 Prosser S. Being patient: Communication and its impact on innovation through the eyes of a patient (and former healthcare senior manager). Int J Healthcare Man. 2010;3:13-30. 